\title{
Influência do tônus muscular na função do membro superior de indivíduos hemiparéticos
}

\author{
Influence of muscle tone on the function of the upper limb \\ of hemiparetic individuals
}

\author{
Influencia del tono muscular en la función de miembro \\ superior de individuos hemipareticos
}

\author{
Geisa Maiara da Silva Feijó ${ }^{1}$, Reni Volmir dos Santos²
}

\begin{abstract}
1.Fisioterapeuta, Graduada, Universidade Luterana do Brasil, ULBRA, Canoas-RS, Brasil.
2.Fisioterapeuta, Mestre, docente do Curso de Fisioterapia da Universidade Luterana do Brasil, ULBRA, Canoas-RS, Brasil.
\end{abstract}

\begin{abstract}
Resumo
Introdução. O Acidente Cerebral Vascular (AVC) é caracterizado por um distúrbio de rápido desenvolvimento, que resulta na restrição da irrigação sanguínea ao cérebro, o que pode levar a disfunções motoras, sensitivas e cognitivas. Por isso, membro superior de indivíduos hemiparéticos tem recebido atenção especial na sua reabilitação, visto que é fundamental para a independência funcional. Objetivo. Avaliar a função do membro superior acometido de indivíduos hemiparéticos. Método. Estudo transversal, realizado na Clínica-escola de Fisioterapia da Universidade Luterana do Brasil, Canoas-RS, com uma amostra de 30 indivíduos com diagnóstico de AVC. Inicialmente preencheram a ficha de identificação, e foram avaliados através da Escala de Tônus Muscular (Ashworth modificada), Escala de Movimentos da Mão, Dinamometria, Escala Motor Activity Log (MAL). Para a associação entre as variáveis, o teste da correlação linear de Spearman foi utilizado, com $<0,05$. Resultados. Predomínio do sexo masculino $(63,3 \%)$, a média de idade foi de $56,3 \pm 13,76$ anos e o tempo de acometimento foi de $49 \pm 63,3$ meses. A correlação do tônus muscular foi significante com as escalas de movimento da mão $(p=0,002)$, MAL quantitativo (qt) $(p=0,006)$, MAL qualitativo (ql) $(p=0,005)$ e com a força de preensão manual $(p=0,047)$. Assim como na correlação de força de preensão manual e escala de movimento da mão com a MAL qt e MAL ql, todas com $p<0,0001$. Conclusão: $O$ tônus muscular influenciou na funcionalidade da mão da amostra estudada, pois quanto mais próximo da eutonia, melhor a sua funcionalidade.
\end{abstract}

Unitermos. Acidente Cerebral Vascular; espasticidade; mão; força muscular

\begin{abstract}
Introduction. Stroke is characterized by a rapid development disorder, which results in the restriction of blood supply to the brain, which can lead to motor, sensitive and cognitive dysfunctions. So the upper limb of hemiparetic individuals has received special attention in their rehabilitation, since it is essential for functional independence. Objective. To evaluate the function of the upper limb affected by hemiparetic individuals. Method. Cross-sectional study, carried out at the Clínica-escola de Fisioterapia da Universidade Luterana do Brasil, Canoas-RS, with a sample of 30 individuals with diagnosis of stroke. Initially, they filled out the identification form, and were evaluated using the Muscle Tone Scale (modified Ashworth), Hand Movement Scale, Dynamometry, Motor Activity Log Scale (MAL). For the association between variables, Spearman's linear correlation test was used, with $<0.05$. Results. Male predominance $(63.3 \%)$, the mean age was $56.3 \pm 13.76$ years and the time of onset was $49 \pm 63.3$ months. The correlation of muscle tone was significant with hand movement scales $(p=0.002)$, quantitative MAL (qt) $(p=0.006)$, qualitative MAL $(q l)(p=0.005)$ and with handgrip strength $(p=0.047)$. As well as in the correlation of handgrip strength and hand movement scale with MAL qt and MAL ql, all with $p<0.0001$. Conclusion. Muscle tone influenced the hand functionality of the studied sample, because the closer to eutony, the better its functionality. Keywords. Stroke; spasticity; hand; muscular strength
\end{abstract}




\begin{abstract}
Resumen
Introducción. El accidente cerebrovascular se caracteriza por un trastorno de rápido desarrollo, que da como resultado la restricción del suministro de sangre al cerebro, lo que puede conducir a trastornos motores, sensibles y cognitivos. Por esta razón, la extremidad superior de los individuos hemiparéticos ha recibido atención especial en su rehabilitación, ya que es esencial para la independencia funcional. Objetivo. Evaluar la función de la extremidad superior afectada de individuos hemiparéticos. Método. Estudio transversal, realizado en la clinica escuela de Fisioterapia de la Universidade Luterana do Brasil. Canoas-RS, con una muestra de 30 individuos diagnosticados de accidente cerebrovascular. Inicialmente completaron el formulario de identificación, y fueron evaluados usando la Escala de tono muscular (Ashworth modificado), Escala de Movimiento de la Mano, Dinamometría, Escala Motor Activity Log (MAL). Para la asociación entre variables, se utilizó la prueba de correlación lineal de Spearman, con $<0.05$. Resultados. Predominio masculino $(63.3 \%)$, la edad promedio fue de $56.3 \pm 13.76$ años y el tiempo de inicio fue de $49 \pm 63.3$ meses. La correlación del tono muscular fue significativa con las escalas de movimiento de la mano $(p=0.002)$, MAL cuantitativa ( $q \mathrm{t})(p=0.006), \operatorname{MAL}$ cualitativa $(q \mathrm{l})(p=0.005)$ y con la fuerza del mango $(p=0,047)$. Así como en la correlación de la fuerza de la empuñadura y la escala del movimiento de la mano con MAL qt y MAL ql, todos con $\mathrm{p}<0.0001$. Conclusión. El tono muscular influyó en la funcionalidad de la mano de la muestra estudiada, porque cuanto más cerca de la eutonía, mejor es su funcionalidad.
\end{abstract}

Palabras clave. accidente cerebrovascular; espasticidad; mano; fuerza muscular

Trabalho realizado na Universidade Luterana do Brasil, Canoas-RS, Brasil

Endereço para correspondência: Reni V Santos. R. Umbu 505/203. CEP 91350-100. Porto Alegre-RS, Brasil. Email: revols@uol.com.br

\title{
INTRODUÇÃO
}

O Acidente Cerebral Vascular (AVC) ocorre por um distúrbio de rápido desenvolvimento, que resulta na restrição da irrigação sanguínea ao cérebro, através da obstrução de uma artéria, caracterizando AVC isquêmico, ou por ruptura de um vaso, no caso do AVC hemorrágico. As causas do AVC isquêmico podem ser por trombose, embolia, dissecação da parede arterial, compressão e malformação, gerando isquemia de áreas focais do cérebro irrigadas pela artéria afetada. A ausência do fluxo sanguíneo gera a necrose dessas áreas que levam a déficits neurológicos, que podem 
ser irreversíveis se as artérias não forem rapidamente desobstruídas. Já o hemorrágico pode ser parenquimatoso ou subaracnóideo. Através da ruptura de uma artéria cerebral, malformação arterial, trauma ou aneurisma, que produz extravasamento sanguíneo por toda região onde houve a lesão, e em muitos casos é necessária a intervenção cirúrgica imediata ${ }^{1,2}$.

De acordo com o modelo da Classificação Internacional de Funcionalidade, os danos após o AVC podem ser descritos como comprometimento da função corporal, diminuição ou perda da função neuromusculoesquelética relacionada ao movimento, mobilidade articular, força muscular, tônus muscular e movimentos involuntários. O córtex motor é o responsável para gerar o impulso do movimento para a medula espinhal, a qual executa o movimento através de sinais para os músculos. Ao ser interrompido, essa transmissão retarda o início e o término da contração muscular, caracterizando as alterações musculares presentes no indivíduo pós-AVC. Deste modo, o membro superior acometido pode apresentar diminuição da força muscular como também apresentar espasticidade 3 .

O membro superior de indivíduos hemiparéticos tem recebido atenção especial na sua reabilitação, visto que, é fundamental para a independência, e as sequelas causadas após o AVC determinam a escolha de instrumentos de avaliação precisos para qualificar e quantificar a funcionalidade, o que permite o planejamento de intervenções terapêuticas devido os grandes obstáculos para 
a reabilitação de indivíduos espásticos ${ }^{4}$. Após o AVC 0 membro superior acometido apresenta dificuldades de realizar movimentos. Sendo que mais de $70 \%$ dos sobreviventes apresentam uma recuperação funcional limitada, e por isso resulta em déficit na qualidade de vida 5 .

As funções desempenhadas pelo membro superior são de extrema importância para a realização das atividades de vida diárias, que inclui o alcance direcionado, a preensão e o manuseio de objetos. A mão humana é um órgão dos sentidos que identifica objetos tamanhos, formas, texturas e consistência dos objetos ${ }^{6}$.

Diante do exposto em relação aos comprometimentos decorrentes do AVC, e sua implicação com o membro superior o presente estudo investigou a correlação do tônus muscular com a funcionalidade do membro superior, através da avaliação da força de preensão manual, movimentos da mão e habilidade motora.

\section{MÉTODO}

\section{Amostra}

Estudo do tipo transversal, realizado no período de agosto a novembro de 2018, na Clínica-escola de Fisioterapia da Universidade Luterana do Brasil, Campus Canoas, Canoas-RS. Esta pesquisa está de acordo com as diretrizes da Resolução 466/12 do Conselho Nacional de Saúde do Ministério da Saúde e foi aprovada pelo Comitê de Ética em Pesquisa da Universidade Luterana do Brasil - Campus de Canoas/RS sob o no 91084818.4.0000.5349. 
Foram incluídos na pesquisa indivíduos hemiparéticos pós-AVC, de ambos os sexos, maiores de 18 anos, totalizando 30 participantes, que assinaram o Termo de Consentimento Livre e Esclarecido.

\section{Procedimento}

Inicialmente os participantes da pesquisa responderam a um questionário de identificação contendo nome, sexo, idade, data do acometimento do AVC e foi apontado o hemicorpo acometido.

Na sequência, os indivíduos foram avaliados através da escala de Ashworth modificada (MAD) que é uma escala subjetiva que avalia o tônus em graus de 0-4. Esta escala classifica o tônus conforme a restrição à movimentação passiva dos grupos musculares de membros superiores e inferiores, graduando conforme a escala, maior o grau, maior o nível da hipertonia (espasticidade) ${ }^{7}$. O sujeito da pesquisa foi orientado em relação ao procedimento realizado em sala desprovida de estímulos visuais ou detratores, posicionado alinhado em decúbito dorsal, em uma maca alta, mantendo seu olhar orientado para cima, não foi permitida sua mobilidade durante o exame. 0 avaliador procedeu à avaliação dos grupos musculares utilizando o posicionamento articular, não utilizando comandos verbais durante cada passo do procedimento. Em um primeiro movimento, foi realizada a mobilização lenta da articulação e um segundo movimento utilizado a mobilização súbita (rápida) da articulação. As mobilizações das articulações 
compreenderam dos seguintes grupos musculares, adutores de ombro, flexores de cotovelo, pronadores, flexores de punho, flexores de dedos e flexores do polegar.

Para avaliação da mobilidade da mão foi utilizada a Escala de Movimentos da Mão (EMM), onde o indivíduo se encontrava sentado, cotovelo fletido a $90^{\circ}$ e antebraço supinado e apoiado, solicitou-se que realizasse a extensão seguida de flexão dos dedos; extensão apenas do segundo dedo, como se estivesse apontando; e pinça entre o primeiro e os demais dedos até chegar a oponência com o dedo mínimo. Esta escala consiste de seis graduações, onde a graduação 1 consiste no fato do indivíduo não realizar nenhum movimento ativo nos dedos, e na graduação 6 há habilidade para realizar a oposição do polegar com todos os dedos $^{8}$.

A avaliação da força muscular (FPM) foi realizada com a utilização da Dinamometria de preensão com o Dinamômetro CAMRY $®$ EH101. A dinamometria foi aplicada para a avaliação da preensão palmar dos participantes, por se tratar de ser uma medida amplamente utilizada como preditora de fraqueza muscular adquirida após o AVC, e podendo, ainda, ser utilizada como indicadora do estado geral da força do paciente. A unidade padrão de medida utilizada foi a de quilograma força (Kgf). Previamente à aplicação do teste, o avaliador demonstrou o procedimento para o paciente e então posicionou o aparelho para a realização da medida. O paciente pressionou o aparelho com a maior contração possível, realizando 3 repetições com 
intervalos de 1 minuto entre elas. O indivíduo esteve sentado confortavelmente, com ombro aduzido, cotovelo fletido a 900, antebraço e punho em posição neutra. Quatro mensurações foram realizadas no membro acometido, sendo a primeira utilizada para adaptação e conhecimento do equipamento, desta forma esta foi descartada, com as demais medidas calculou-se a média aritmética ${ }^{9}$.

A avaliação da habilidade motora foi realizada através da Escala Motor Activity Log (MAL), versão portuguesa, traduzida e adaptada. A MAL tem o formato de entrevista semi-estruturada, na qual os indivíduos são requisitados a avaliar o uso real do membro superior (MS) mais afetado em atividades manuais, dentro do seu contexto de vida. Inicialmente, os indivíduos foram esclarecidos sobre a finalidade do instrumento, e enfatizado a diferença entre as duas escalas, quantitativa e qualitativa do uso do MS mais afetado nas atividades. Se o indivíduo não tiver utilizado o MS mais afetado para alguma atividade, é possível analisar e classificar o motivo por meio de um registro de codificação ${ }^{10}$.

Assim, a MAL deve ser aplicada sob a forma de entrevista, e pode ser realizada com o indivíduo ou com o seu cuidador, engloba duas escalas ordinais, com seis pontos em cada, para a graduação das atividades: uma relacionada à quantidade de uso e outra à qualidade do uso. Considerando a escala quantitativa, a pontuação varia de "não usa o MS mais afetado" (pontuação zero) a "usa o MS mais afetado da mesma forma que usava antes da história 
de AVC" (pontuação cinco). Para a escala qualitativa, a pontuação varia de "o MS mais afetado não é usado de forma alguma para a atividade" (pontuação zero) a "sua habilidade de usar o MS mais afetado é tão boa quanto era antes do AVC" (pontuação cinco). A pontuação total é obtida com o cálculo da média para cada uma das escalas. Quanto maior a média obtida nas escalas, melhor a qualidade e quantidade de uso do MS mais afetado na realização das atividades de vida diária (AVD). Uma grande vantagem da MAL é que na situação em que alguma atividade não se aplica ao indivíduo, o item pode ser descartado e o cálculo da média pode ser refeito considerando os itens restantes ${ }^{10}$.

\section{Análise Estatística}

Após os procedimentos, os dados foram alocados em planilha Excel e analisados, no programa SPSS versão 21.0, através do coeficiente de correlação de Spearman. A magnitude das correlações foi baseada na classificação de Munro (baixa=0,26-0,49; moderada=0,50-0,69; alta=0,700,89; muito alta $=0,90-1,00)$ para interpretação dos coeficientes de correlação. O nível de significância foi estabelecido em $p \leq 0,05$.

\section{RESULTADOS}

A caracterização da amostra do presente estudo encontra-se na Tabela 1, sendo que a idade e tempo de AVC estão expressos em média e desvio padrão. Já o sexo, número de indivíduos e lado de acometimento está descrito 
conforme a incidência. A faixa etária dos participantes compreende de 22 a 79 anos, sendo que a maioria apresenta idade acima dos 50 anos, com uma prevalência de $70 \%$.

Tabela 1. Caracterização da amostra.

\begin{tabular}{ll}
\hline Característica & Incidência \\
\hline Sexo & 19 homens \\
& 11 mulheres \\
\hline Idade & $56,3 \pm 13,76$ \\
\hline Tempo médio de AVC & $49,0 \pm 63,30$ \\
\hline Hemicorpo acometido & Direito: 18 \\
& Esquerdo: 12 \\
\hline
\end{tabular}

Os valores referentes ao grau do tônus dos participantes estão descritos na Tabela 2, conforme a avaliação dos músculos, assim como também está expresso a graduação total do tônus do membro superior acometido. Também se encontram os escores das escalas MAL (quantitativo e qualitativo) e EMM. Já os valores da FPM estão expressos pela unidade $\mathrm{kg} / \mathrm{f}$.

A correlação da graduação de tônus pela MAD com MAL, EMM e FPM está demonstrada na Tabela 3. Os resultados demonstram correlação significante.

Já a correlação entre as escalas MAL, EMM e FPM está descrita na Tabela 4. Conforme se observa, todas as correlações foram significantes. 
Tabela 2. Escore das escalas Ashworth Modificada, de Movimento da Mão, Motor Activity Log (quantitativo e qualitativo) e Força de Preensão Manual (kg/f).

\begin{tabular}{ll}
\hline Escalas & Média (DP) \\
\hline Escala de Ashworth modificada & \\
Adutores do ombro & $1,0 \pm 0,96$ \\
Flexores de cotovelo & $1,1 \pm 0,98$ \\
Pronadores & $0,7 \pm 0,96$ \\
Flexores do punho & $0,9 \pm 0,90$ \\
Flexores dos dedos & $0,9 \pm 0,99$ \\
Flexores do polegar & $0,8 \pm 1,00$ \\
Todo membro superior & $0,9 \pm 0,86$ \\
\hline Motor Activity Log (quantitativo) & $41,4 \pm 45,04$ \\
\hline & $40,8 \pm 44,13$ \\
\hline Motor Activity Log (qualitativo) & \\
\hline Escala de Movimentos da Mão & $4,1 \pm 2,23$ \\
\hline Força de Preensão Manual & $12,3 \pm 9,06$ \\
\hline DP: desvio-padrão.
\end{tabular}

Tabela 3. Correlação da escala de graduação do tônus de Ashworth Modificada (MAD) com a Escala de Movimento da Mão (EMM), escala Motor Activity Log quantitativo e qualitativo (MALqt e MALql) e força de preensão manual (FPM) através do coeficiente de correlação de Spearman $\left(r_{s}\right)$.

\begin{tabular}{lccc}
\hline Correlação & rs & Classificação & p \\
\hline MAD - EMM & 0,548 & Moderada & 0,002 \\
MAD - FPM & 0,543 & Moderada & 0,047 \\
MAD - MALqt & 0,494 & Baixa & 0,006 \\
MAD - MALql & 0,498 & Baixa & 0,005 \\
\hline
\end{tabular}

Tabela 4. Correlação das escalas de escala de movimento da mão (EMM), Motor Activity Log quantitativo e qualitativo (MALqt e MALql) e força de preensão manual (FPM) através do coeficiente de correlação de Spearman $\left(r_{s}\right)$.

\begin{tabular}{llcc}
\hline Correlação & rs & Classificação & p \\
\hline FPM - MALqt & 0,768 & Alta & $<0,0001$ \\
FPM - MALql & 0,767 & Alta & $<0,0001$ \\
EMM - FPM & 0,712 & Alta & $<0,0001$ \\
EMM - MALqt & 0,788 & Alta & $<0,0001$ \\
EMM - MALql & 0,792 & Alta & $<0,0001$ \\
\hline
\end{tabular}




\section{DISCUSSÃO}

A média de idade, deste estudo, foi um pouco abaixa do observado na literatura, numa amostra de 23 indivíduos, foi observada uma média de $61,69 \pm 15,43$ anos, com a faixa etária de 36 a 77 anos $^{4}$. Semelhante a outro estudo, com uma amostra de 32 indivíduos, em que a média de idade foi de $61,59 \pm 15,50$ anos $^{11}$. Em outro estudo com 25 pacientes também foi encontrada uma média similar, sendo esta de 65 anos, com faixa etária entre 37 e 92 anos, demonstrando a prevalência de pacientes com idade superior a 50 anos $^{12}$.

Em relação ao sexo, o presente estudo assemelha-se a outro, duplo cego, com 26 pacientes pós-AVC, sendo 9 mulheres $(34,62 \%)$ e 17 homens $(65,38 \%)^{13}$. Outro estudo de caráter exploratório, quantitativo, descritivo do tipo transversal com 18 pacientes, realizou a pesquisa entre maio e agosto de 2013 e foram encontrados valores semelhantes sendo 11 homens $(61,11 \%)$ e 7 mulheres $(38,89 \%)^{14}$. Numa amostra de 30 indivíduos, também predominou o gênero masculino $(66,6 \%)$ para $(33,4 \%)$ do gênero feminino ${ }^{15}$. Ao investigarem os episódios de AVC, em uma área urbana da Croácia, foi identificado, numa amostra de 396 indivíduos, $210(53,04 \%)$ mulheres e $186(46,96 \%)$ homens ${ }^{16}$. Em um estudo piloto realizado na clínica escola de fisioterapia da faculdade Maurício de Nassau em Teresina-PI, foi observado em 21 indivíduos, 13 eram mulheres e 8 eram homens ${ }^{17}$. Apesar de a literatura apontar um predomínio quanto ao gênero mais propenso do acometimento de AVC, nota-se que 
não há um consenso, o que pode supor que ambos os sexos estão predispostos.

Quanto ao tempo de acometimento do AVC encontrouse uma faixa de 2 a 240 meses, sendo a média de $49 \pm 63,3$ meses. No estudo, com uma amostra de 7 pacientes, sendo 3 homens e 4 mulheres, foi observada uma média de tempo de lesão de 66,14 meses $^{18}$. Em outro estudo com participação de 13 pacientes de centro de reabilitação e do Hospital Universitário de São Carlos, São Paulo, apresentaram um tempo médio de lesão de 75,23 meses $^{19}$. Numa amostra de 18 indivíduos, sendo 11 homens e 7 mulheres, em um estudo no Centro de Reabilitação de Lesão Cerebral, Copenhague, Dinamarca, observaram indivíduos com um média de 39 meses de tempo de $\mathrm{AVC}^{20}$. Nos estudos verificam-se diferentes tempos de lesão, demonstrando a cronicidade dos pacientes, semelhante ao presente estudo que apresenta uma média de 49 meses de lesão.

$\mathrm{Na}$ correlação do tônus muscular com a força de preensão manual, respectivamente pela escala de Ashworth modificada e a dinamometria, pode-se notar, que quanto mais próximo o tônus se encontra da eutonia, maior a força muscular do membro superior acometido. Trinta e dois indivíduos pós-AVC tratados com vibração mecânicoacústica, por meio de um dispositivo $\operatorname{Viss} \AA, 3$ vezes por semana, num total 12 sessões, em musculatura do membro superior $^{11}$, foi verificado que o tônus passou de $1,59 \pm 1,33$ para $1,12 \pm 1,05$, pela escala de Ashworth, assim como houve aumento da força de preensão palmar, onde os valores 
passaram de $13,88 \pm 8,88 \mathrm{kgf}$ para $17,24 \pm 10,11 \mathrm{kgf}$, no grupo interventivo $(n=17)$, porém no grupo controle $(n=15)$, que só realizou simulação, tanto o tônus quanto a força muscular de preensão manual se mantiveram inalteradas. Apesar deste estudo não ser interventivo, pode-se observar esta correlação do tônus muscular com a força muscular.

Ao analisar a correlação da força muscular com a função motora foram avaliados 77 pacientes, sendo 33 pacientes subagudos e 44 pacientes crônicos, quanto a força global, força de preensão manual e função motora ${ }^{21}$. Verificaram a correlação da força muscular, sendo esta avaliada de duas maneiras, através da dinamometria e do uso do esfigmomanômetro, e a função motora pela escala de FuglMeyer. Em ambos os casos observou-se uma correlação significante da funcionalidade com a força sendo que, quanto maior a força muscular melhor a funcionalidade $(p<0,0001)$. Assim nota-se que os resultados dos autores supracitados assemelham-se com este estudo, em que foi observada a mesma correlação.

$\mathrm{Na}$ verificação do tônus muscular com a habilidade motora, respectivamente pela $M A D$ e pela $M A L$, pode-se notar, ao correlacionar os dois instrumentos, que quanto mais próximo o tônus se encontra da eutonia, melhor a habilidade motora do membro superior acometido. Neste intuito, foram avaliados 33 pacientes pós-AVC, com idade média de 61,5 anos e com média de tempo de duração do AVC de 37,7 meses $^{22}$. O estudo utilizado foi clínico randomizado, sendo os pacientes submetidos a 12 sessões 
de estimulação somatossensorial e treinamento específico da tarefa (TST). Os pacientes realizaram 2 horas de atividade somatossensorial ativa ou estimulação simulada seguida por 30 minutos de TST, 3 vezes por semana por 4 semanas. Após a intervenção, foram reavaliados com 3 e 6 meses, não apresentado alteração nesses intervalos das avaliações, e a correlação entre a MALqt e a MAD se manteve significante $(p=0,0,01)$ em todo esse período.

Um estudo experimental clínico randomizado longitudinal e prospectivo, com uma amostra de 27 indivíduos divididos em grupo controle com 10 pacientes e grupo intervenção com 17 pacientes $^{23}$, usando a realidade virtual para reabilitação de pacientes pós-AVC, que foram submetidos a um protocolo de exercícios com cinco sessões semanais, de 1 hora e 15 minutos durante duas semanas, totalizando 10 sessões. Este estudo também correlacionou a função motora através da escala de Fugl-Meyer (FM) e a habilidade motora através da escala Motor Activity Log $(M A L)$, onde se verificou que houve aumento do escore na comparação da avaliação com a reavaliação. Para a escala de Fugl Meyer $(p=0,0001)$, e para a escala MAL $(p=0,0001)$. Porém, não houve diferenças intergrupos para $\operatorname{FM}(p=0,10)$ e $\operatorname{MAL}(0,30)$.

Houve correlação entre a força de preensão muscular, através da dinamometria, com a mobilidade motora, pela escala de movimento da mão, e com o tônus muscular, pela escala de Ashworth, em 43 pacientes, sendo 18 mulheres e 25 homens, com idade média de $60,7 \pm 12,1$ anos e tempo 
pós-AVC de 22 meses $^{24}$. Os resultados da correlação da força com o movimento da mão foram significantes $(p=0,0072)$, porém não foi observada correlação com o tônus muscular $(p=0,21)$. Neste, conforme apresentado na Tabela 3, também apresentou uma correlação significante, assim como observado na tabela ao comparar a força com o tônus. Tal diferença entre os dois trabalhos pode estar nos graus de espasticidade entre as amostras, pois mesmo as duas escalas caracterizando como leve, o estudo supracitado não apresenta nos seus resultados o desvio padrão, somente a média do tônus, que foi de 0,7 . Pois com ele dá para ter uma melhor ideia da dispersão dos valores da amostra. Mesmo assim, indicam que os resultados melhores na motricidade manual (força e movimento) sugerem menor grau de espasticidade.

Num estudo realizado no ambulatório de Fisioterapia, submeteram 16 pacientes, com idades na faixa etária de 31 a 75 anos, divididos em grupo controle e grupo intervenção ${ }^{25}$, utilizando a Terapia de Movimento Induzido pela Restrição (TMIR) através de uma tipoia no membro superior não comprometido, enquanto os pacientes do grupo controle realizaram 0 mesmo treino funcional. Foram realizadas dez sessões, em duas semanas consecutivas, de segunda a sexta-feira, durante quatro horas diárias, e os pacientes foram avaliados antes, pós-terapia e após os 3 meses. Foram utilizados para as avaliações instrumentos padronizados com a finalidade de avaliar a função motora do membro superior, que são a MAL, o Wolf Motor Function Test 
(WMFT), o Action Research Arm Test (ARAT), estes últimos verificam as atividades do membro superior através de quatro tipos básicos de função: compressão, preensão, pinçamento e atividades de alcance.

Apesar do estudo não apresentar uma correlação entre os testes, pode-se observar em ambos os grupos que o valor médio da MAL, este estava em concordância com os testes WMFT e do ARAT ${ }^{25}$. E quando no grupo intervenção houve aumento do escore da MAL, os escores dos outros testes também aumentaram, e no controle os valores médios se mantiveram. Assim estes achados estão coerentes com os encontrados neste estudo, pois os participantes que tinham um melhor escore da MAL apresentavam uma melhor mobilidade da mão. Num estudo randomizado, realizado em cinco hospitais de Taiwan entre 2012 e 2014, com 39 pacientes divididos em 2 grupos, um para intervenção de terapia assistida por robô associado com estimulação elétrica neuromuscular e o outro grupo de terapia assistida por robô e estimulação simulada, por um período de 90 a 100 minutos, durante 5 dias da semana por 4 semanas $^{26}$. Foram avaliados através das escalas: Fugl-Meyer da extremidade superior (UE-FMA), escala de Ashworth modificada, WMFT, MAL e Stroke Impact Scale 3.0. Os resultados do WMFT-FAS (capacidade funcional) revelaram uma interação grupoxtempo $(p=0,019)$ e um efeito principal de tempo $(p<0,001)$. Ambos os grupos após a intervenção demonstraram ser significantes na avaliação da qualidade e 
quantidade de movimento através da MALql $(p<0,001)$ e qt $(p<0,001)$.

Nem estudo randomizado em dois grupos, um com terapia de restrição de movimento induzida e um grupo controle, com uma amostra de 13 pacientes ${ }^{27}$, o grupo intervenção recebeu tratamento durante 2 horas por dia, 5 dias por semanas durante 3 semanas consecutivas e o grupo controle recebeu reabilitação tradicional compatível com o grupo intervenção em duração e intensidade. O estudo apresentou, após o tratamento, um aumento para o grupo intervenção com uma maior eficácia que o grupo controle na escala de Fugl-Meyer $(p=0,04)$, MALqt $(p=0,02)$ e MALql $(p=0,02)$. Assim, os estudos anteriores reforçam os achados encontrados nesse estudo, pois se observa a correlação dos escores da MAL com a funcionalidade do membro superior.

\section{CONCLUSÃO}

Através dos resultados foi possível observar, na amostra estudada, que o tônus influencia na função do membro superior de indivíduos hemiparéticos. Sendo assim, quanto maior o grau do tônus (espasticidade), maior a incapacidade de executar movimentos, falta de habilidade motora e perda de qualidade e quantidade de movimento do membro acometido.

Contudo, por se tratar de uma pesquisa que contempla a variação no tempo de cronicidade, o conhecimento desses parâmetros pode fornecer subsídios importantes no que 
tange à importância de uma avaliação específica do membro superior acometido para essa população. Porém novos trabalhos devem ser realizados através da investigação de outros itens de avaliação, para qualificar mais a necessidade da atenção para o uso do membro afetado pós-AVC.

\section{REFERÊNCIAS}

1.Kusumaningsih W, Rachmayanti S, Werdhani RA. Relationship between risk factors and activities of daily living using modified Shah Barthel Index in stroke patients. J Phys 2017;884:1-17. http://dx.doi.org/10.1088/1742-6596/884/1/012151

2.Amarenco P, Lavallée PC, Labreuche J, Albers GW, Bornstein NM, Canho $\mathrm{P}$, et al. One-year risk of stroke after transient ischemic attack or minor stroke. N Engl J Med 2016;374:1533-42. http://dx.doi.org/10.1056/NEJMoa1412981

3. Raghavan P. Upper limb motor impairment after stroke. Phys Med Rehabil Clin N Am 2015;26:599-610.

http://dx.doi.org/10.1016/j.pmr.2015.06.008

4.Constantino C, Galuppo L, Romiti D. Efficacy of Mechano-Acoustic Vibration on Strength, Pain, and Function in Post Stroke Rehabilitation: A Pilot Study. Top Stroke Rehabil 2014;21:391-9. http://dx.doi.org/10.1310/tsr2105-391

5. Lannin NA, Cusick A, Hills C, Kinnear B, Vogel K, Matthews K, et al. Upper limb motor training using a Saebo TM orthosis is feasible for increasingtask-specific practice in hospital after stroke. Aus Occupat Therap J 2016;63:364-72. http://dx.doi.org/10.1111/14401630.12330

6. Wattchow KA, McDonnell MN, Hillier SL. Rehabilitation interventions for upper limb function in the first four weeks following stroke: a systematic review and meta-analysis of the evidence. Arc Phys Med Rehab 2017;99:367-82.

http://dx.doi.org/10.1016/j.apmr.2017.06.014

7.Pascual-Pascual SI, Herrera-Galante A, Póo P, García-Aymerich V, Aguilar-Barberà M, Bori-Fortuny I, et al. Guidelines for the treatment of child spasticity using botulinum toxin. Rev Neurol 2007;44:303-9. http://dx.doi.org/10.33588/rn.4405.2006340.

8. Soares AV, Kerscher C, Uhlig L, Domenech SC, Junior NGB. Escala de movimentos da mão: um instrumento preditivo da recuperação funcional do membro superior de pacientes hemiparéticos por Acidente Vascular Cerebral. Arq Catar Med 2011;4047-51. http://dx.doi.org/10.590/1809-2950/14143922042015

9.Araujo BF, Nascimento CM, Busarello FO, Moreira NB, Baroni MP, Carvalho AR, et al. Avaliação da força de preensão palmar frente à 
terapia com mobilização neural. Rev Bras Med Esporte 2012;18:2425. http://dx.doi.org/10.1590/S1517-86922012000400005

10.Saliba VA, Magalhães LC, Faria CDCM, Laurentino GEC, Cassiano JG, Teixeira-Salmela LF. Adaptação transcultural e análise das propriedades psicométricas da versão brasileira do instrumento Motor Activity Log. Rev Panam Salud Publica 2011;30:262-71. http://dx.doi.org/10.1590/S1020-49892011000900011

11.Constantino C, Galuppo L, Romiti D. Short-term effect of local muscle vibration treatment versus sham therapy on upper limb in chronic post-stroke patients: a randomized controlled Trial Cosimo. Eur J Phys Rehab Med 2017;53:32-40. http://dx.doi.org/10.23736/S19739087.16.04211-8

12.Grabherr L, Jola C, Berra G, Robert Theiler R, Mast FW. Motor imagery training improves precision of na upper limb movement in patients with hemiparesis. NeuroRehabilitation 2015;36,157-66. http://dx.doi.org/10.3233/NRE-151203

13.Ili'c NV, Raspopovi'c ED, Nedeljkovi'c U, Vujadinovi'c ST, Milanovi'c SD, Markovi'c IP, et al. Effects of na odalt DCSando ccupation al therapy on fine motor skill deficits in patients with chronic stroke. Restor Neurol Neurosci 2016;34:935-45.

http://dx.doi.org/10.1016/j.jped.2013.05.010

14.Sá ES, Filho ACS, Jesus SC, Lima FCVM. Força de preensão palmar através da dinamometria em indivíduos hemiparéticos pós acidente vascular encefálico. Rev Bras Prescr Fisiol Exer 2017;11:180-6. http://www.rbpfex.com.br/index.php/rbpfex/article/view/1074

15.Pereira ND, Ovando AC, Michaelsen SM, Dos Anjos SM, Lima RCM, Nascimento LR, et al. Motor Activity Log-Brasil: confiabilidade e relações com a função motora em indivíduos com acidente vascular cerebral crônico. Arq Neuropsiqu 2012;70:196-201. http://dx.doi.org/10.1590/S0004-282X2012000300008

16. Kes VB, Jurašić MJ, Zavoreo I, Jelec V, Matovina LZ. Age and gender differences in acute stroke hospital patients. Acta Clin Croat 2016;55:69-78. http://dx.doi.org/10.20471/acc.2016.55.01.11

17. Oliveira MCB, Silva DRC, Cortez BV, Coêlho CKS, Silva FMS, Oliveira GBVP, et al. Mirror and Vibration Therapies Effects on the Upper Limbs of Hemiparetic Patients after Stroke: A Pilot Study. Rehabil Res Chand Pract 2018;2018:6183654. http://dx.doi.org/10.1155/2018/6183654 18. Oliveira JNM, Lima ES, Mamede CAGS, Santos WVS, Marinho RF, Duarte PHM, et al. Avaliação funcional de pacientes acometidos pelo acidente vascular encefálico e submetidos à terapia de contensão induzida. Arch Health Invest 2018;7:408-14. http://dx.doi.org/10.2127/archi.v7i10.3169

19.Santos GL, Souza MB, Desloovere K, Russo TL. Elastic Tape Improved Shoulder Joint Position Sense in Chronic Hemiparetic Subjects: A Randomized Sham-Controlled Crossover Study. PLoS ONE 2017;12:1-17. http://dx.doi.org/10.1371/journal.pone.0170368

20.Vinstrup J, Calatayud J, Jakobsen MD, Sundstrup E, Jorgensen JR, Casaña J, et al. Hand strengthening exercises in chronic stroke 
patients: Dose-response evaluation using electromyography. J Hand Ther 2017;31:111-21. http://dx.doi.org/10.1016/j.jht.2017.01.004 21. Martins JC, Aguiar LT, Lara EM, Moura JB, Souza LAC, TeixeiraSalmela LF, et al. Assessment of grip strength with the modified sphygmomanometer test: association between upper limb global strengt hand motor function. Fisiot Mov 2015;9:498-506. http://dx.doi.org/10.1590/0103-5150.029.001.AR04

22.Fleming MK, Sorinola IO, Lewis SFR, Wolfe CD, Wellwood I, Newham DJ. The Effect of Combined Somatosensory Stimulation and Task-Specific Training on Upper Limb Function in Chronic Stroke: A Double-Blind Randomized Controlled Trial. Neurorehabil Neural Repair 2014;29:143-52. http://dx.doi.org/10.1177/1545968314533613

23. Galvão MLC, Gouvêa PM, Ocamoto GN, Silva AT, Reis LM, Kosour C, et al. Efeito da Realidade Virtual na Função Motora do Membro Superior Parético Pós-Acidente Vascular Cerebral. Rev Neurocienc 2015;23:493-8. http://dx.doi.org/10.4181/RNC.2015.23.04.1038.06p 24.Soares AV, Kerscher C, Uhlig L, Domenech SC, Junior NGB. Dinamometria de preensão manual como parâmetro de avaliação funcional do membro superior de pacientes hemiparéticos por acidente vascular cerebral. Fisioter Pes 2011;18:359-64.

http://dx.doi.org/10.1590/S1809-29502011000400011

25. Almeida AS, Santos JS, Garção DC. Análise da consolidação da funcionalidade manual de hemiparéticos crônicos após terapia de movimento induzido pela restrição. Rev Ter Ocup Univ São Paulo 2016;27:297-304.

http://dx.doi.org/10.11606/issn.22386149.v27i3p297-304

26.Lee Y-Y, Lin K-C, Cheng H-J, Wu C-C, Hsieh Y-W, Chen C-K. Effects of combining robot-assisted therapy with neuromuscular electrical stimulation on motor impairment, motor and daily function, and quality of life in patients with chronic stroke: a double-blinded randomized controlled trial. J Neuro Eng Rehab 2015;12:96. http://dx.doi.org/10.1186/s12984-015-0088-3.

27. Lin K-C, Chung H-Y, Wu C-Y, Liu H-L, Hsieh Y-W, Chen I-H, et al. Constraint-Induced Therapy Versus Control Intervention in Patients with Stroke. A Functional Magnetic Resonance Imaging Study. Am J Phys Med Rehabil 2010;89:177-85. http://dx.doi.org/10.1097/PHM.0b013e3181cf1c78 